\title{
Tests used to evaluate dizziness in primary care
}

\author{
Jacquelien Dros MD, Otto R. Maarsingh MD, Henriëtte E. van der Horst MD PhD, \\ Patrick J. Bindels MD PhD, Gerben ter Riet MD PhD, Henk C. van Weert MD PhD
}

Previously published at www.cmaj.ca

$\mathrm{D}$ izziness is a nonspecific term that refers to various abnormal sensations of body orientation in space; patients often find these sensations difficult to describe. The prevalence of dizziness in the community ranges from $1.8 \%$ among young adults to over $30 \%$ among the elderly. Yearly consultation rates because of dizziness in primary care ranges from $2.5 \%$ among patients aged 25 to 44 years, to $8.3 \%$ among patients aged 65 years and older, and to $18.2 \%$ among patients aged 85 years and older. ${ }^{1-5}$

Dizziness can be classified into four subtypes: vertigo, disequilibrium, presyncope and atypical dizziness. This classification is based on the study by Drachman and Hart. ${ }^{6}$ There are few studies of the distribution of the specific diseases that can cause dizziness. The following data should, therefore, be interpreted with caution (Appendix 1, available at www.cmaj.ca/cgi/content /full/cmaj.080910/DC1). ${ }^{5.7-11}$ In primary care patients, vertigo (prevalence $38 \%$ ) is mainly caused by otolaryngical conditions; disequilibrium (prevalence 10\%) is mainly caused by orthopedic, neurologic or sensory problems; presyncope (prevalence 10\%) is mainly caused by cardiac or vasomotor conditions; and atypical dizziness (prevalence 17\%) is mainly caused by psychiatric problems. In about $25 \%$ of patients, the type cannot be classified or the problem has multiple causes.

The prevalence of these diagnostic categories differs according to age. In younger patients, atypical dizziness and presyncope are most common. In middle-aged patients, vertigo is the most prominent. Presyncope and disequilibrium are more prevalent in elderly patients..$^{5,12,13}$ However, elderly patients' symptoms cannot always be placed in one category because their symptoms often have multiple causes. ${ }^{5,12,13}$

Practice guidelines ${ }^{14-16}$ advocate the use of several diagnostic tests in the evaluation of dizziness, including historytaking, pulse measurement, carotid sinus massage, nystagmus tests and the Dix-Hallpike manoeuvre. However, these recommendations are based more on opinion than on evidence.

Many tests can only be performed in secondary and tertiary care settings, although most patients are first seen in primary care. The main problem for primary care physicians is to decide which patients need additional testing, which should be referred to secondary care, which require immediate therapy, and which should receive an explanation, reassurance, advice and a "wait and see" approach.

Life-threatening conditions requiring immediate treatment are rare in patients with dizziness (Appendix 2, available at www.cmaj.ca/cgi/content/full/cmaj.080910/DC1). ${ }^{3,6,8,17}$ In

\section{Key points}

- Studies on diagnosing dizziness have been conducted in highly selected homogeneous groups of patients only.

- Evidence to support the diagnostic process in primary care is scarce.

- An exception is the head impulse test, with a positive test result being diagnostic of peripheral vestibular dysfunction and a negative test result diagnostic of central peripheral dysfunction.

these mainly acute conditions (mastoiditis, pacemaker failure, myocardial or brainstem/cerebellar infarction, hemorrhage and serious electrolyte disturbances), dizziness is almost never the only presenting symptom, and obtaining a careful patient history and performing a targeted physical examination is usually sufficient for triage. Further diagnostic testing should be done in a secondary care setting. Primary care physicians need to know the characteristics of the diagnostic tests that can be used as point-of-care tests for the diagnosis of the more common conditions.

We performed a systematic review of diagnostic tests that can be used to diagnose dizziness in patients in primary care. Because prevalence is important in the discriminative power of such tests, we provide epidemiologic information about target conditions in patients who present with dizziness. We have limited our review to the evaluation of these tests in patients with dizziness.

\section{Methods}

\section{Literature search}

We searched MEDLINE, EMBASE, PsycINFO, CINAHL, and GeroLit from database inception to May 2009 to identify studies of diagnostic tests for dizziness in primary care. The complete search strategy is shown in Appendix 3 (available at www.cmaj.ca/cgi/content/full/cmaj.080910/DC1). We used the search filters reported by Bachmann and colleagues. ${ }^{18,19}$

From the Department of Family Medicine (Dros, ter Riet, van Weert), Academic Medical Center, University of Amsterdam, Amsterdam; the Department of Family Medicine (Maarsingh, van der Horst), EMGO-institute, VU Medical Center, Amsterdam; the Department of Family Medicine (Bindels), Erasmus Medical Center, Erasmus University Rotterdam, Rotterdam, the Netherlands.

CMAJ 2010. DOI:10.1503/cmaj.080910 


\section{Study selection}

Two reviewers (J.D., O.M.) independently selected potentially relevant studies based on titles and abstracts. If disagreement occurred, consensus was reached with a third reviewer (H.vW. or H.vdH.). We excluded studies if the title did not include "dizziness," "disequilibrium," "(pre)syncope" or "vertigo" or a word with the same meaning, or if it did not involve a disease that can cause dizziness (Appendix 3). We excluded articles if the abstract did not mention any diagnostic procedure, if the study population did not include patients with dizziness or if the test studied was too complex, timeconsuming or expensive to be feasible in primary care (e.g., carotid sinus massage, tilt table testing, posturography and specialized imaging techniques). We included only studies published in English, French, German or Dutch.

\section{Data extraction and quality assessment}

The methodological quality of the studies was assessed by two pairs of reviewers (O.M. and H.vdH. or J.D. and H.vW.) using the Quality Assessment of Diagnostic Accuracy Studies (QUADAS) checklist. ${ }^{20}$ Within each pair, both reviewers independently scored all studies with the QUADAS checklist, extracted the data and constructed $2 \times 2$ tables. Consensus was reached for any discrepancies. If an article reported the

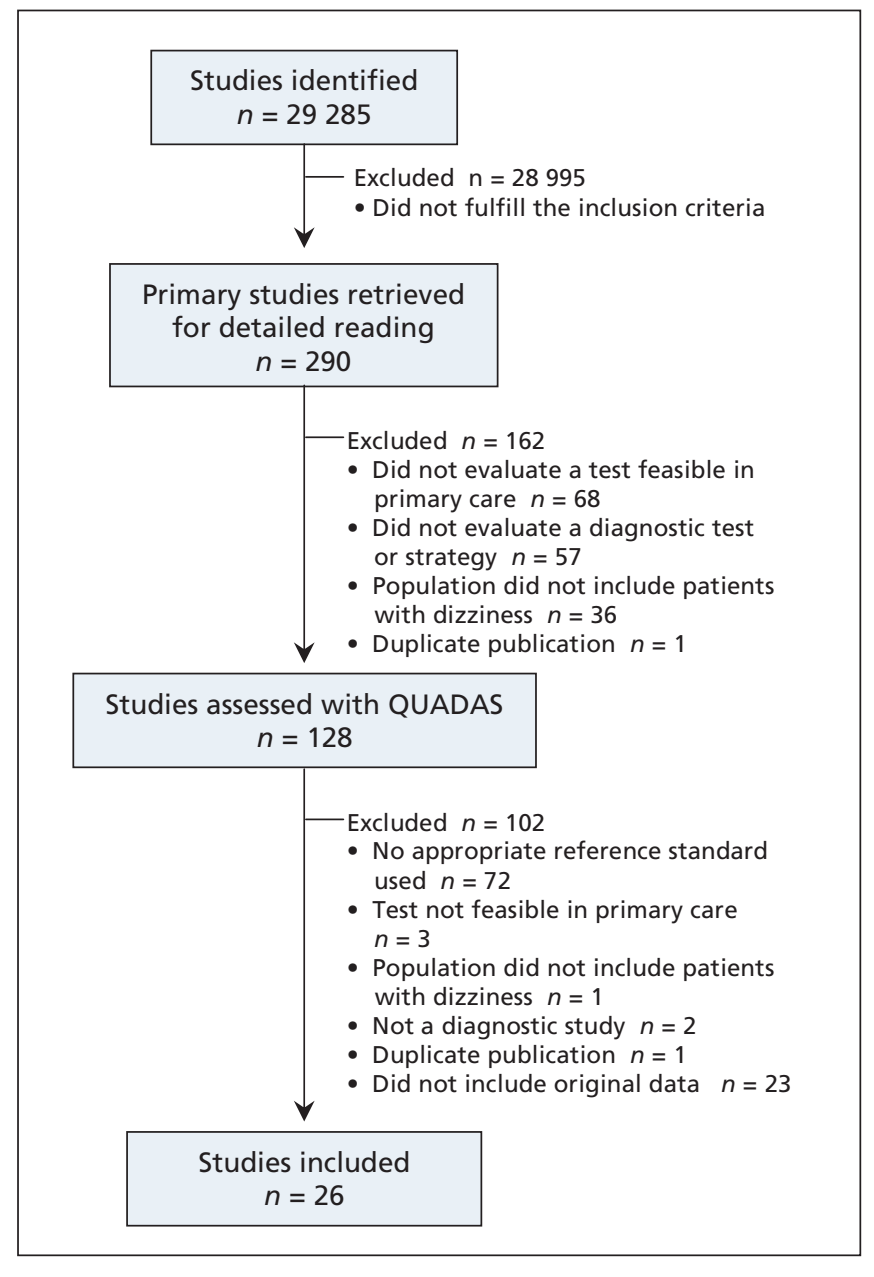

Figure 1: Flow chart of the study selection process. accuracy of more than one index test, target condition or cutoff point, we report the data for these tests, target conditions and cut-off points separately.

\section{Data synthesis and analysis}

We calculated sensitivity and specificity from the $2 \times 2$ tables and plotted the results in forest plots and receiver operating characteristics plots. We pooled the results from studies involving the same tests and conditions by use of a bivariate regression model that takes into account the correlation between sensitivity and specificity. This method was recently recommended for meta-analysis of diagnostic tests. ${ }^{21,22}$ If we could only extract the sensitivity of the test from the original data, we calculated the mean sensitivity and weighted it for the number of patients in the study. To assess clinical usefulness, we derived likelihood ratios and pre- and post-test probabilities of the target condition in the study population.

Because we were interested in the accuracy of the diagnostic tests in primary care but most of the studies were done in secondary or tertiary care settings, we calculated pre- and post-test probabilities for a hypothetical primary care situation, assuming that the sensitivity and specificity would not be different in primary care. We estimated and retrieved the pretest probabilities of the target conditions in primary care from pertinent prevalence studies in primary care (Appendix 1).

We used STATA/SE 10.1 (StataCorp, College Station, TX, USA) to perform the bivariate analyses.

\section{Results}

The study selection process is shown in Figure 1. We identified 290 primary articles that studied diagnostic tests that can be used in the primary care of patients with dizziness. The kappa statistic was 0.81 for agreement on study selection by the two reviewers (J.D., O.M.). Based on a review of the full text, we excluded 162 articles. We assessed 128 articles using QUADAS. ${ }^{20}$ After this review, we excluded 102 articles (Figure 1). Thus, we included 26 articles in this review (Table 1). ${ }^{23-48}$ We included one study of a psychiatric test (subtype atypical), and 25 studies of neuro-otologic tests (subtype vertigo). No studies about disequilibrium and presyncope were available. Five studies involved two or more tests, five studies reported on target conditions, and two studies evaluated two cut-off points of the same test (Table 2).

\section{Methodologic quality}

Quality assessment with the QUADAS tool is presented in Appendix 4 (available at www.cmaj.ca/cgi/content /full/cmaj.080910/DC1). Each study had at least two methodologic limitations that could introduce bias. In almost all studies, the spectrum of included patients was not representative of primary care patients.

\section{Sensitivity, specificity and likelihood ratios}

Table 2 shows the sensitivity, specificity and positive and negative likelihood ratios of the included studies. The preand post-test probabilities of the target condition in the study 
Table 1: Characteristics of 26 studies of diagnostic tests of dizziness feasible in primary care (part 1)

\begin{tabular}{|c|c|c|c|c|c|c|}
\hline Study & $\begin{array}{c}\text { No. of } \\
\text { participants } \\
\text { (\% women) }\end{array}$ & $\begin{array}{l}\text { Age, yr, } \\
\text { mean } \\
\text { (range) }\end{array}$ & $\begin{array}{l}\text { Study entry and } \\
\text { inclusion criteria }\end{array}$ & Setting & Target condition & $\begin{array}{l}\text { Index test; } \\
\text { reference standard }\end{array}$ \\
\hline Persoons et al. ${ }^{43}$ & $97(66)$ & $\begin{array}{c}48 \\
(18-75)\end{array}$ & $\begin{array}{l}\text { Random sample of } \\
\text { dizzy patients } \\
\text { referred to } \\
\text { otorhinolaryngologist }\end{array}$ & $\begin{array}{l}\text { Outpatient tertiary } \\
\text { academic clinic, } \\
\text { Belgium }\end{array}$ & $\begin{array}{l}\text { Major depressive } \\
\text { disorder; panic } \\
\text { disorder }\end{array}$ & $\begin{array}{l}\text { Depression and panic } \\
\text { disorder parts of the patient } \\
\text { health questionnaire; MINI } \\
\text { interview }\end{array}$ \\
\hline \multicolumn{7}{|l|}{ Questionnaires } \\
\hline *Kentala and Rauch ${ }^{36}$ & $57(74)$ & $\begin{array}{c}47 \\
(20-78)\end{array}$ & $\begin{array}{l}\text { Consecutive patients } \\
\text { with dizziness }\end{array}$ & $\begin{array}{l}\text { Outpatient tertiary } \\
\text { clinic, US }\end{array}$ & Vertigo & $\begin{array}{l}\text { Dizziness questionnaire; } \\
\text { diagnosis after clinical } \\
\text { evaluation }\end{array}$ \\
\hline López-Escámez et al. ${ }^{37}$ & $100(65)$ & NR & $\begin{array}{l}\text { Nonselected patients } \\
\text { with dizziness or } \\
\text { vertigo }\end{array}$ & $\begin{array}{l}\text { Outpatient clinic, } \\
\text { Spain }\end{array}$ & Ménière disease & $\begin{array}{l}\text { Structured questionnaire of } \\
\text { vertigo; Ménière Disease } \\
\text { criteria of the American } \\
\text { Academy of } \\
\text { Otolaryngology, Head and } \\
\text { Neck Surgery; } 10 \text { months } \\
\text { follow-up }\end{array}$ \\
\hline von Brevern et al. ${ }^{24}$ & $61(51)$ & $\begin{array}{l}N R \\
(18 \text { to } \\
>60)\end{array}$ & $\begin{array}{l}\text { Consecutive patients } \\
\text { with dizziness }\end{array}$ & $\begin{array}{l}\text { Outpatient tertiary } \\
\text { clinics, Germany }\end{array}$ & BPPV & $\begin{array}{l}\text { Vestibular vertigo } \\
\text { questionnaire; Dix-Hallpike } \\
\text { manoeuver and/or diagnosis } \\
\text { after clinical evaluation }\end{array}$ \\
\hline Whitney et al. ${ }^{48}$ & $373(64)$ & $\begin{array}{c}61 \\
(15-95)\end{array}$ & $\begin{array}{l}\text { Nonselected patients } \\
\text { with balance and/or } \\
\text { vestibular disorder }\end{array}$ & $\begin{array}{l}\text { Outpatient } \\
\text { academic (physical } \\
\text { therapy) clinic, US }\end{array}$ & BPPV & $\begin{array}{l}\text { DHI BPPV subscore, } 5 \text { items } \\
\text { or } 2 \text { items; Dix-Hallpike } \\
\text { manoeuver }\end{array}$ \\
\hline \multicolumn{7}{|l|}{ Symptoms and signs } \\
\hline $\mathrm{Chan}^{27}$ & $88(52)$ & $\begin{array}{c}79 \\
\text { (median) } \\
(33-90)\end{array}$ & $\begin{array}{l}\text { Consecutive patients } \\
\text { with dizziness }\end{array}$ & $\begin{array}{l}\text { Outpatient tertiary } \\
\text { clinic, China }\end{array}$ & BPPV & $\begin{array}{l}\text { Spinning; episodic; } \\
\text { positional; nausea/vomiting; } \\
\text { Dix-Hallpike and side-lying } \\
\text { manoeuver }\end{array}$ \\
\hline Norré $^{41}$ & $85(61)$ & $\begin{array}{c}54 \\
(14-87)\end{array}$ & $\begin{array}{l}\text { Selected patients } \\
\text { with vertigo on head } \\
\text { movement }\end{array}$ & $\begin{array}{l}\text { Outpatient } \\
\text { academic clinic, } \\
\text { Belgium }\end{array}$ & BPPV & Battery of vestibular tests \\
\hline \multicolumn{7}{|l|}{ Side-lying manoeuver } \\
\hline Cohen $^{29}$ & 29 (NR) & NR & $\begin{array}{l}\text { Selected patients } \\
\text { with vertigo on head } \\
\text { movement and } \\
\text { referred for } \\
\text { vestibular testing }\end{array}$ & $\begin{array}{l}\text { Outpatient } \\
\text { tertiary academic } \\
\text { clinic, US }\end{array}$ & BPPV & Battery of vestibular tests \\
\hline \multicolumn{7}{|c|}{ Head-shaking nystagmus } \\
\hline Burgio et al..$^{25}$ & 115 (NR) & NR & $\begin{array}{l}\text { Nonselected referred } \\
\text { dizzy patients }\end{array}$ & $\begin{array}{l}\text { Outpatient } \\
\text { academic clinic, US }\end{array}$ & $\begin{array}{l}\text { Peripheral } \\
\text { vestibular } \\
\text { dysfunction }\end{array}$ & Caloric tests and ENG \\
\hline
\end{tabular}


Table 1: Characteristics of 26 studies of diagnostic tests of dizziness feasible in primary care (part 2)

\begin{tabular}{|c|c|c|c|c|c|c|}
\hline Study & $\begin{array}{c}\text { No. of } \\
\text { participants } \\
\text { (\% women) }\end{array}$ & $\begin{array}{c}\text { Age, } \\
\text { yr, } \\
\text { mean } \\
\text { (range) }\end{array}$ & $\begin{array}{l}\text { Study entry and } \\
\text { inclusion criteria }\end{array}$ & Setting & Target condition & $\begin{array}{l}\text { Index test; } \\
\text { reference standard }\end{array}$ \\
\hline Fujimoto et al. ${ }^{30}$ & $1364(58)$ & $\begin{array}{c}48 \\
(8-91)\end{array}$ & $\begin{array}{l}\text { Consecutive patients } \\
\text { during routine ENG } \\
\text { testing }\end{array}$ & $\begin{array}{l}\text { Outpatient } \\
\text { academic clinic, } \\
\text { Canada }\end{array}$ & $\begin{array}{l}\text { Peripheral } \\
\text { vestibular } \\
\text { dysfunction }\end{array}$ & $\begin{array}{l}>20 \% \text { difference in right/left } \\
\text { caloric testing }\end{array}$ \\
\hline Guidetti et al. ${ }^{32}$ & $661(65)$ & $\begin{array}{c}55 \\
(14-80)\end{array}$ & $\begin{array}{l}\text { Nonselected patients } \\
\text { with and without } \\
\text { vertigo }\end{array}$ & $\begin{array}{l}\text { Outpatient tertiary } \\
\text { academic clinic, } \\
\text { Italy }\end{array}$ & $\begin{array}{l}\text { Peripheral } \\
\text { vestibular } \\
\text { dysfunction }\end{array}$ & Caloric tests \\
\hline Harvey et al. ${ }^{34}$ & $105(67)$ & $\begin{array}{c}52 \\
(13-87)\end{array}$ & $\begin{array}{l}\text { Consecutive dizzy } \\
\text { patients }\end{array}$ & $\begin{array}{l}\text { Outpatient tertiary } \\
\text { academic clinic, US }\end{array}$ & $\begin{array}{l}\text { Peripheral } \\
\text { vestibular } \\
\text { dysfunction }\end{array}$ & Caloric tests \\
\hline Mandala et al..$^{38}$ & $65(37)$ & $\begin{array}{c}54 \\
(13-84)\end{array}$ & $\begin{array}{l}\text { Selected patients } \\
\text { with vestibular } \\
\text { neuritis }\end{array}$ & $\begin{array}{l}\text { Outpatient } \\
\text { academic clinic, } \\
\text { Italy }\end{array}$ & $\begin{array}{l}\text { Peripheral } \\
\text { vestibular } \\
\text { dysfunction } \\
\text { (vestibular } \\
\text { neuritis) }\end{array}$ & Caloric tests \\
\hline Tseng and $\mathrm{Chao}^{45}$ & $258(62)$ & $\begin{array}{c}\text { NR } \\
(14-79)\end{array}$ & $\begin{array}{l}\text { Consecutive patients } \\
\text { with dizziness or } \\
\text { vertigo }\end{array}$ & $\begin{array}{l}\text { Outpatient } \\
\text { academic clinic, } \\
\text { Taiwan }\end{array}$ & $\begin{array}{l}\text { Peripheral } \\
\text { vestibular } \\
\text { dysfunction }\end{array}$ & $\begin{array}{l}\text { Caloric tests } \\
\text { with ENG }\end{array}$ \\
\hline Vicini et al. ${ }^{46}$ & $277(53)$ & $\begin{array}{c}47 \\
(6-83)\end{array}$ & $\begin{array}{l}\text { NR; Referred patients } \\
\text { with vestibular } \\
\text { and/or neurological } \\
\text { complaints }\end{array}$ & $\begin{array}{l}\text { Outpatient } \\
\text { academic clinics, } \\
\text { Italy }\end{array}$ & $\begin{array}{l}\text { Peripheral and } \\
\text { central vestibular } \\
\text { dysfunction }\end{array}$ & Battery of vestibular tests \\
\hline Harvey et al..$^{33}$ & $\begin{array}{c}112(65) \\
(224 \text { ears) }\end{array}$ & $\begin{array}{c}53 \\
(13-87)\end{array}$ & NR; dizzy patients & $\begin{array}{l}\text { Outpatient } \\
\text { academic clinic, US }\end{array}$ & $\begin{array}{l}\text { Peripheral } \\
\text { vestibular } \\
\text { dysfunction }\end{array}$ & Caloric tests \\
\hline Harvey et al. ${ }^{34}$ & $105(67)$ & $\begin{array}{c}52 \\
(13-87)\end{array}$ & $\begin{array}{l}\text { Consecutive dizzy } \\
\text { patients }\end{array}$ & $\begin{array}{l}\text { Outpatient tertiary } \\
\text { academic clinic, US }\end{array}$ & $\begin{array}{l}\text { Peripheral } \\
\text { vestibular } \\
\text { dysfunction }\end{array}$ & Caloric tests \\
\hline Mandala et al..$^{38}$ & $65(37)$ & $\begin{array}{c}54 \\
(13-84)\end{array}$ & $\begin{array}{l}\text { Selected patients } \\
\text { with vestibular } \\
\text { neuritis }\end{array}$ & $\begin{array}{l}\text { Outpatient } \\
\text { academic clinic, } \\
\text { Italy }\end{array}$ & $\begin{array}{l}\text { Peripheral } \\
\text { vestibular } \\
\text { dysfunction } \\
\text { (vestibular } \\
\text { neuritis) }\end{array}$ & Caloric tests \\
\hline
\end{tabular}


Table 1: Characteristics of 26 studies of diagnostic tests of dizziness feasible in primary care (part 3)

\begin{tabular}{|c|c|c|c|c|c|c|}
\hline Study & $\begin{array}{c}\text { No. of } \\
\text { participants } \\
\text { (\% women) }\end{array}$ & $\begin{array}{l}\text { Age, } \\
\text { yr, } \\
\text { mean } \\
\text { (range) }\end{array}$ & $\begin{array}{l}\text { Study entry and } \\
\text { inclusion criteria }\end{array}$ & Setting & Target condition & $\begin{array}{l}\text { Index test; } \\
\text { reference standard }\end{array}$ \\
\hline $\begin{array}{l}\text { Newman-Toker } \\
\text { et al. }{ }^{40}\end{array}$ & $42(35)$ & $\begin{array}{c}64 \\
(26-92)\end{array}$ & $\begin{array}{l}\text { Consecutive referred } \\
\text { patients with acute } \\
\text { vertigo and high risk } \\
\text { for stroke }\end{array}$ & $\begin{array}{l}\text { Outpatient tertiary } \\
\text { academic clinic, US }\end{array}$ & $\begin{array}{l}\text { Peripheral } \\
\text { (vestibular } \\
\text { neuritis) and } \\
\text { central vestibular } \\
\text { dysfunction } \\
\text { (brainstem or } \\
\text { cerebellar stroke) }\end{array}$ & $\begin{array}{l}\text { ENG or } \\
\text { videonystagmography and } \\
\text { cranial MRI }\end{array}$ \\
\hline $\begin{array}{l}\text { Perez and Rama- } \\
\text { Lopez }^{42}\end{array}$ & $265(50)$ & $\begin{array}{c}50 \\
(N R)\end{array}$ & $\begin{array}{l}\text { Nonselected referred } \\
\text { patients with vertigo }\end{array}$ & $\begin{array}{l}\text { Outpatient } \\
\text { academic clinic, } \\
\text { Spain }\end{array}$ & $\begin{array}{l}\text { Peripheral } \\
\text { vestibular } \\
\text { dysfunction }\end{array}$ & Caloric tests \\
\hline \multicolumn{7}{|c|}{ Vibration-induced nystagmus } \\
\hline Mandala et al..$^{38}$ & $64(37)$ & $\begin{array}{c}54 \\
(13-84)\end{array}$ & $\begin{array}{l}\text { Selected patients } \\
\text { with vestibular } \\
\text { neuritis }\end{array}$ & $\begin{array}{l}\text { Outpatient } \\
\text { academic clinic, } \\
\text { Italy }\end{array}$ & $\begin{array}{l}\text { Peripheral } \\
\text { vestibular } \\
\text { dysfunction } \\
\text { (vestibular } \\
\text { neuritis) }\end{array}$ & Caloric tests \\
\hline Michel et al. ${ }^{39}$ & 399 (NR) & NR & $\begin{array}{l}\text { NR; vertiginous } \\
\text { patients and } \\
\text { nonvertiginous } \\
\text { patients }\end{array}$ & $\begin{array}{l}\text { Outpatient clinics, } \\
\text { France }\end{array}$ & $\begin{array}{l}\text { Peripheral } \\
\text { vestibular } \\
\text { dysfunction }\end{array}$ & Caloric tests \\
\hline \multicolumn{7}{|c|}{ Cold mini caloric test } \\
\hline $\begin{array}{l}\text { Weinberg } \\
\text { and Sadé }{ }^{47}\end{array}$ & 124 (NR) & NR & $\begin{array}{l}\text { NR; patients with } \\
\text { vestibular neuronitis, } \\
\text { Ménière disease, } \\
\text { acoustic tumor or } \\
\text { dizziness }\end{array}$ & $\begin{array}{l}\text { Outpatient clinic, } \\
\text { Israel }\end{array}$ & $\begin{array}{l}\text { Peripheral } \\
\text { vestibular } \\
\text { dysfunction }\end{array}$ & Clinical diagnosis \\
\hline \multicolumn{7}{|l|}{ Head heave test } \\
\hline Mandala et al. ${ }^{38}$ & $65(37)$ & $\begin{array}{c}54 \\
(13-84)\end{array}$ & $\begin{array}{l}\text { Selected patients } \\
\text { with vestibular } \\
\text { neuritis }\end{array}$ & $\begin{array}{l}\text { Outpatient } \\
\text { academic clinic, } \\
\text { Italy }\end{array}$ & $\begin{array}{l}\text { Peripheral } \\
\text { vestibular } \\
\text { dysfunction } \\
\text { (vestibular } \\
\text { neuritis) }\end{array}$ & Caloric tests \\
\hline \multicolumn{7}{|c|}{ High-frequency oscillopsia test } \\
\hline Burgio et al. ${ }^{26}$ & 115 (NR) & NR & $\begin{array}{l}\text { Nonselected referred } \\
\text { dizzy patients }\end{array}$ & $\begin{array}{l}\text { Outpatient } \\
\text { academic clinic, US }\end{array}$ & $\begin{array}{l}\text { Peripheral } \\
\text { vestibular } \\
\text { dysfunction }\end{array}$ & Caloric tests and ENG \\
\hline \multicolumn{7}{|c|}{ Hyperventilation-induced nystagmus } \\
\hline Robichaud et al. ${ }^{44}$ & $38(66)$ & $\begin{array}{c}53 \\
(25-72)\end{array}$ & $\begin{array}{l}\text { Selected referred } \\
\text { dizzy patients with } \\
>25 \% \text { difference in } \\
\text { Right/left caloric } \\
\text { testing }\end{array}$ & $\begin{array}{l}\text { Outpatient tertiary } \\
\text { academic clinic, } \\
\text { Canada }\end{array}$ & $\begin{array}{l}\text { Peripheral } \\
\text { vestibular } \\
\text { dysfunction }\end{array}$ & Caloric tests \\
\hline Robichaud et al..$^{44}$ & $24(54)$ & $\begin{array}{c}58 \\
(39-78)\end{array}$ & $\begin{array}{l}\text { Patients with acoustic } \\
\text { neurinoma }\end{array}$ & $\begin{array}{l}\text { Outpatient tertiary } \\
\text { academic clinic, } \\
\text { Canada }\end{array}$ & $\begin{array}{l}\text { Central vestibular } \\
\text { dysfunction }\end{array}$ & Cranial MRI \\
\hline \multicolumn{7}{|c|}{ Positional nystagmus test } \\
\hline Guidetti et al. ${ }^{32}$ & $661(65)$ & $\begin{array}{c}55 \\
(14-80)\end{array}$ & $\begin{array}{l}\text { Nonselected patients } \\
\text { with and without } \\
\text { vertigo }\end{array}$ & $\begin{array}{l}\text { Outpatient tertiary } \\
\text { academic clinic, } \\
\text { Italy }\end{array}$ & $\begin{array}{l}\text { Peripheral } \\
\text { vestibular } \\
\text { dysfunction }\end{array}$ & Caloric tests \\
\hline
\end{tabular}


Table 2: Data from 26 studies that tested the accuracy of diagnostic tests for diagnosing dizziness in primary care (part 1)

\begin{tabular}{|c|c|c|c|c|c|c|c|c|}
\hline Study & $\begin{array}{l}\text { True- } \\
\text { positive } \\
\text { results }\end{array}$ & $\begin{array}{l}\text { False- } \\
\text { positive } \\
\text { results }\end{array}$ & $\begin{array}{l}\text { False- } \\
\text { negative } \\
\text { results }\end{array}$ & $\begin{array}{l}\text { True- } \\
\text { negative } \\
\text { results }\end{array}$ & $\begin{array}{l}\text { Sensitivity } \\
(95 \% \mathrm{Cl})\end{array}$ & $\begin{array}{l}\text { Specificity } \\
(95 \% \mathrm{Cl})\end{array}$ & $\begin{array}{l}\text { LR of positive } \\
\text { result }(95 \% \mathrm{Cl})\end{array}$ & $\begin{array}{l}\text { LR of negative } \\
\text { result }(95 \% \mathrm{Cl})\end{array}$ \\
\hline \multicolumn{9}{|c|}{ PSYCHIATRY, PSYCHOGENIC DIZZINESS } \\
\hline \multicolumn{9}{|c|}{ Target condition = major depressive disorder } \\
\hline \multicolumn{9}{|c|}{ Patient health questionnaire } \\
\hline Persoons et al. ${ }^{43}$ & 8 & 5 & 3 & 81 & $\begin{array}{c}72.7 \\
(0.39-0.94)\end{array}$ & $\begin{array}{c}94.2 \\
(0.87-0.98)\end{array}$ & $\begin{array}{c}12.51 \\
(4.96-31.53)\end{array}$ & $\begin{array}{c}0.29 \\
(0.11-0.76)\end{array}$ \\
\hline \multicolumn{9}{|c|}{ Target condition = panic disorder } \\
\hline \multicolumn{9}{|c|}{ Patient health questionnaire } \\
\hline Persoons et al. ${ }^{43}$ & 16 & 3 & 1 & 77 & $\begin{array}{c}94.1 \\
(0.71-1.00)\end{array}$ & $\begin{array}{c}96.3 \\
(0.89-0.99)\end{array}$ & $\begin{array}{c}25.1 \\
(8.22-76.65)\end{array}$ & $\begin{array}{c}0.06 \\
(0.01-0.41)\end{array}$ \\
\hline \multicolumn{9}{|l|}{ NeURo-Otology } \\
\hline \multicolumn{9}{|c|}{ Target condition = benign paroxysmal positional vertigo } \\
\hline \multicolumn{9}{|l|}{ Questionnaires } \\
\hline von Brevern et al. ${ }^{24}$ & 22 & 3 & 3 & 33 & $\begin{array}{c}88.0 \\
(0.69-0.98)\end{array}$ & $\begin{array}{c}91.7 \\
(0.78-0.98)\end{array}$ & $\begin{array}{c}10.56 \\
(3.54-31.5)\end{array}$ & $\begin{array}{c}0.13 \\
(0.05-0.38)\end{array}$ \\
\hline $\begin{array}{l}\text { Whitney et al. }{ }^{48} ; \mathrm{DHI} \\
\text { BPPV subscore, } \\
5 \text { items }\end{array}$ & 62 & 179 & 22 & 110 & $\begin{array}{c}73.8 \\
(0.63-0.83)\end{array}$ & $\begin{array}{c}38.1 \\
(0.32-0.44)\end{array}$ & $\begin{array}{c}1.19 \\
(1.02-1.39)\end{array}$ & $\begin{array}{c}0.69 \\
(0.47-1.01)\end{array}$ \\
\hline $\begin{array}{l}\text { Whitney et al. }{ }^{48} ; \mathrm{DHI} \\
\text { BPPV subscore, } \\
2 \text { items }\end{array}$ & 34 & 71 & 50 & 218 & $\begin{array}{c}40.5 \\
(0.30-0.52)\end{array}$ & $\begin{array}{c}75.4 \\
(0.70-0.80)\end{array}$ & $\begin{array}{c}1.65 \\
(1.19-2.29)\end{array}$ & $\begin{array}{c}0.79 \\
(0.65-0.95)\end{array}$ \\
\hline \multicolumn{9}{|l|}{ Symptoms and signs } \\
\hline $\begin{array}{l}\text { Chann }^{27} \text {; spinning } \\
\text { sensation }\end{array}$ & 3 & 30 & 2 & 53 & $\begin{array}{c}60.0 \\
(0.15-0.95)\end{array}$ & $\begin{array}{c}63.9 \\
(0.53-0.74)\end{array}$ & $\begin{array}{c}1.66 \\
(0.77-3.59)\end{array}$ & $\begin{array}{c}0.63 \\
(0.21-1.86)\end{array}$ \\
\hline $\begin{array}{l}\text { Chan }{ }^{27} \text {; episodic } \\
\text { attacks of dizziness }\end{array}$ & 4 & 61 & 1 & 22 & $\begin{array}{c}80.0 \\
(0.28-1.00)\end{array}$ & $\begin{array}{c}26.5 \\
(0.17-0.37)\end{array}$ & $\begin{array}{c}1.09 \\
(0.69-1.72)\end{array}$ & $\begin{array}{c}0.76 \\
(0.13-4.52)\end{array}$ \\
\hline $\begin{array}{l}\text { Chan }^{27} \text {; rapid head } \\
\text { movements } \\
\text { provoking dizziness }\end{array}$ & 4 & 53 & 1 & 30 & $\begin{array}{c}80.0 \\
(0.28-1.00)\end{array}$ & $\begin{array}{c}36.1 \\
(0.26-0.47)\end{array}$ & $\begin{array}{c}1.25 \\
(0.79-2.00)\end{array}$ & $\begin{array}{c}0.55 \\
(0.09-3.27)\end{array}$ \\
\hline $\begin{array}{l}\text { Chan }^{27} \text {; associated } \\
\text { with nausea and/or } \\
\text { vomiting }\end{array}$ & 1 & 30 & 4 & 53 & $\begin{array}{c}20.0 \\
(0.01-0.72)\end{array}$ & $\begin{array}{c}63.9 \\
(0.53-0.74)\end{array}$ & $\begin{array}{c}0.55 \\
(0.09-3.27)\end{array}$ & $\begin{array}{c}1.25 \\
(0.79-2.00)\end{array}$ \\
\hline \multicolumn{9}{|c|}{ Dix-Hallpike manoeuver } \\
\hline Cohen $^{29}$ & 17 & & 12 & & $\begin{array}{c}58.6 \\
(0.39-0.77)\end{array}$ & & & \\
\hline Norréé & 74 & & 11 & & $\begin{array}{c}87.1 \\
(0.78-0.93)\end{array}$ & & & \\
\hline \multicolumn{9}{|l|}{ Side-lying manoeuver } \\
\hline Cohen $^{29}$ & 19 & & 10 & & $\begin{array}{c}65.5 \\
(0.46-0.82)\end{array}$ & & & \\
\hline \multicolumn{9}{|c|}{ Target condition = vertigo } \\
\hline \multicolumn{9}{|l|}{ Questionnaire } \\
\hline Kentala and Rauch ${ }^{36}$ & 21 & 8 & 14 & 14 & $\begin{array}{c}60.0 \\
(0.42-0.76)\end{array}$ & $\begin{array}{c}63.6 \\
(0.44-0.84)\end{array}$ & $\begin{array}{c}1.65 \\
(0.89-3.05)\end{array}$ & $\begin{array}{c}0.63 \\
(0.38-1.05)\end{array}$ \\
\hline \multicolumn{9}{|c|}{ Target condition = Ménière disease } \\
\hline \multicolumn{9}{|l|}{ Questionnaire } \\
\hline $\begin{array}{l}\text { López-Escámez } \\
\text { et al. }{ }^{37}\end{array}$ & 5 & 2 & 1 & 92 & $\begin{array}{c}83.3 \\
(0.36-1.00)\end{array}$ & $\begin{array}{c}97.9 \\
(0.93-1.00)\end{array}$ & $\begin{array}{c}39.17 \\
(9.5-161.55)\end{array}$ & $\begin{array}{c}0.17 \\
(0.03-1.02)\end{array}$ \\
\hline
\end{tabular}




\begin{tabular}{|c|c|c|c|c|c|c|c|c|}
\hline Study & $\begin{array}{l}\text { True- } \\
\text { positive } \\
\text { results }\end{array}$ & $\begin{array}{l}\text { False- } \\
\text { positive } \\
\text { results }\end{array}$ & $\begin{array}{l}\text { False- } \\
\text { negative } \\
\text { results }\end{array}$ & $\begin{array}{l}\text { True- } \\
\text { negative } \\
\text { results }\end{array}$ & $\begin{array}{l}\text { Sensitivity } \\
(95 \% \mathrm{Cl})\end{array}$ & $\begin{array}{l}\text { Specificity } \\
(95 \% \mathrm{Cl})\end{array}$ & $\begin{array}{l}\text { LR of positive } \\
\text { result }(95 \% \mathrm{Cl})\end{array}$ & $\begin{array}{l}\text { LR of negative } \\
\text { result }(95 \% \mathrm{Cl})\end{array}$ \\
\hline \multicolumn{9}{|c|}{ Target condition = peripheral vestibular dysfunction } \\
\hline \multicolumn{9}{|c|}{ Head-shaking nystagmus } \\
\hline Burgio et al. ${ }^{25}$ & 15 & 39 & 20 & 41 & $\begin{array}{c}42.9 \\
(0.26-0.61)\end{array}$ & $\begin{array}{c}51.2 \\
(0.40-0.63)\end{array}$ & $\begin{array}{c}0.88 \\
(0.56-1.37)\end{array}$ & $\begin{array}{c}1.11 \\
(0.78-1.59)\end{array}$ \\
\hline Fujimoto et al. ${ }^{30}$ & 130 & 302 & 129 & 803 & $\begin{array}{c}50.2 \\
(0.44-0.56)\end{array}$ & $\begin{array}{c}72.7 \\
(0.70-0.75)\end{array}$ & $\begin{array}{c}1.84 \\
(1.57-2.14)\end{array}$ & $\begin{array}{c}0.69 \\
(0.60-0.78)\end{array}$ \\
\hline Goebel and Garcia ${ }^{31}$ & 18 & 26 & 25 & 145 & $\begin{array}{c}41.9 \\
(0.27-0.58)\end{array}$ & $\begin{array}{c}84.8 \\
(0.79-0.90)\end{array}$ & $\begin{array}{c}2.75 \\
(1.67-4.54)\end{array}$ & $\begin{array}{c}0.69 \\
(0.53-0.89)\end{array}$ \\
\hline Guidetti et al. ${ }^{32}$ & 106 & 13 & 422 & 120 & $\begin{array}{c}20.1 \\
(0.17-0.24)\end{array}$ & $\begin{array}{c}90.2 \\
(0.84-0.95)\end{array}$ & $\begin{array}{c}2.05 \\
(1.19-3.54)\end{array}$ & $\begin{array}{c}0.89 \\
(0.83-0.95)\end{array}$ \\
\hline Harvey et al. ${ }^{34}$ & 7 & 7 & 13 & 78 & $\begin{array}{c}35.0 \\
(0.15-0.59)\end{array}$ & $\begin{array}{c}91.8 \\
(0.84-0.97)\end{array}$ & $\begin{array}{c}4.25 \\
(1.68-10.74)\end{array}$ & $\begin{array}{c}0.71 \\
(0.51-0.98)\end{array}$ \\
\hline Jacobson et al..$^{35}$ & 23 & 1 & 28 & 64 & $\begin{array}{c}45.1 \\
(0.31-0.60)\end{array}$ & $\begin{array}{c}98.5 \\
(0.92-1.00)\end{array}$ & $\begin{array}{c}29.31 \\
(4.10-209.83)\end{array}$ & $\begin{array}{c}0.56 \\
(0.43-0.72)\end{array}$ \\
\hline Mandala et al. ${ }^{38}$ & 62 & & 3 & & $\begin{array}{c}95.4 \\
(0.87-0.99)\end{array}$ & & & \\
\hline Tseng and $\mathrm{Chao}^{45}$ & 74 & 82 & 8 & 94 & $\begin{array}{c}90.2 \\
(0.82-0.96)\end{array}$ & $\begin{array}{c}53.4 \\
(0.46-0.61)\end{array}$ & $\begin{array}{c}1.94 \\
(1.63-2.30)\end{array}$ & $\begin{array}{c}0.18 \\
(0.09-0.36)\end{array}$ \\
\hline Vicini et al. ${ }^{46}$ & 31 & 42 & 60 & 144 & $\begin{array}{c}34.1 \\
(0.24-0.45)\end{array}$ & $\begin{array}{c}77.4 \\
(0.71-0.83)\end{array}$ & $\begin{array}{c}1.51 \\
(1.02-2.23)\end{array}$ & $\begin{array}{c}0.85 \\
(0.72-1.01)\end{array}$ \\
\hline \multicolumn{9}{|l|}{ Head impulse test } \\
\hline Beynon et al. ${ }^{23}$ & 25 & 0 & 26 & 99 & $\begin{array}{c}49.0 \\
(0.35-0.63)\end{array}$ & $\begin{array}{c}100 \\
(0.96-1.00)\end{array}$ & $\begin{array}{c}98.08 \\
(6.09-1578.88)\end{array}$ & $\begin{array}{c}0.51 \\
(0.39-0.67)\end{array}$ \\
\hline Cnyrim et al. ${ }^{28}$ & 37 & 17 & 3 & 26 & $\begin{array}{c}92.5 \\
(0.80-0.98)\end{array}$ & $\begin{array}{c}60.5 \\
(0.44-0.75)\end{array}$ & $\begin{array}{c}2.34 \\
(1.60-3.42)\end{array}$ & $\begin{array}{c}0.12 \\
(0.04-0.38)\end{array}$ \\
\hline Harvey et al. ${ }^{33}$ & 13 & 6 & 20 & 185 & $\begin{array}{c}39.4 \\
(0.23-0.58)\end{array}$ & $\begin{array}{c}96.9 \\
(0.93-0.99)\end{array}$ & $\begin{array}{c}12.54 \\
(5.13-30.66)\end{array}$ & $\begin{array}{c}0.63 \\
(0.47-0.82)\end{array}$ \\
\hline Harvey et al. ${ }^{34}$ & 7 & 4 & 13 & 81 & $\begin{array}{c}35.0 \\
(0.15-0.59)\end{array}$ & $\begin{array}{c}95.3 \\
(0.88-0.99)\end{array}$ & $\begin{array}{c}7.44 \\
(2.41-22.97)\end{array}$ & $\begin{array}{c}0.68 \\
(0.49-0.94)\end{array}$ \\
\hline Mandala et al. ${ }^{38}$ & 54 & & 11 & & $\begin{array}{c}83.1 \\
(0.72-0.91)\end{array}$ & & & \\
\hline $\begin{array}{l}\text { Newman-Toker } \\
\text { et al. }{ }^{40}\end{array}$ & 8 & 3 & 0 & 31 & $\begin{array}{c}100.0 \\
(0.63-1.00)\end{array}$ & $\begin{array}{c}91.2 \\
(0.76-0.98)\end{array}$ & $\begin{array}{c}9.44 \\
(3.45-25.84)\end{array}$ & $\begin{array}{c}0.06 \\
(0.00-0.91)\end{array}$ \\
\hline $\begin{array}{l}\text { Perez and Rama- } \\
\text { Lopez }^{42} ; \text { asymmetry } \\
\text { caloric test }>22 \%\end{array}$ & 79 & 12 & 60 & 114 & $\begin{array}{c}56.8 \\
(0.48-0.65)\end{array}$ & $\begin{array}{c}90.5 \\
(0.84-0.95)\end{array}$ & $\begin{array}{c}5.97 \\
(3.42-10.42)\end{array}$ & $\begin{array}{c}0.48 \\
(0.39-0.58)\end{array}$ \\
\hline $\begin{array}{l}\text { Perez and Rama- } \\
\text { Lopez }{ }^{42} \text {; asymmetry } \\
\text { caloric test }>42.5 \%\end{array}$ & 71 & 20 & 21 & 153 & $\begin{array}{c}77.2 \\
(0.67-0.85)\end{array}$ & $\begin{array}{c}88.4 \\
(0.83-0.93)\end{array}$ & $\begin{array}{c}6.68 \\
(4.36-10.20)\end{array}$ & $\begin{array}{c}0.26 \\
(0.18-0.38)\end{array}$ \\
\hline \multicolumn{9}{|c|}{ Vibration-induced nystagmus } \\
\hline Mandala et al. ${ }^{38}$ & 52 & & 12 & & $\begin{array}{c}81.3 \\
(0.70-0.90)\end{array}$ & & & \\
\hline Michel et al. ${ }^{39}$ & 45 & 32 & 6 & 316 & $\begin{array}{c}88.2 \\
(0.76-0.96)\end{array}$ & $\begin{array}{c}90.8 \\
(0.87-0.94)\end{array}$ & $\begin{array}{c}9.6 \\
(6.8-13.55)\end{array}$ & $\begin{array}{c}0.13 \\
(0.06-0.28)\end{array}$ \\
\hline \multicolumn{9}{|l|}{ Cold mini caloric test } \\
\hline $\begin{array}{l}\text { Weinberg and } \\
\text { Sadé }^{47}\end{array}$ & 51 & 8 & 3 & 62 & $\begin{array}{c}94.4 \\
(0.85-0.99)\end{array}$ & $\begin{array}{c}88.6 \\
(0.79-0.95)\end{array}$ & $\begin{array}{c}8.26 \\
(4.29-15.91)\end{array}$ & $\begin{array}{c}0.06 \\
(0.02-0.19)\end{array}$ \\
\hline \multicolumn{9}{|l|}{ Head heave test } \\
\hline Mandala et al. ${ }^{38}$ & 43 & & 22 & & $\begin{array}{c}66.2 \\
(0.53-0.77)\end{array}$ & & & \\
\hline
\end{tabular}




\begin{tabular}{|c|c|c|c|c|c|c|c|c|}
\hline Study & $\begin{array}{l}\text { True- } \\
\text { positive } \\
\text { results }\end{array}$ & $\begin{array}{l}\text { False- } \\
\text { positive } \\
\text { results }\end{array}$ & $\begin{array}{l}\text { False- } \\
\text { negative } \\
\text { results }\end{array}$ & $\begin{array}{l}\text { True- } \\
\text { negative } \\
\text { results }\end{array}$ & $\begin{array}{l}\text { Sensitivity } \\
(95 \% \mathrm{Cl})\end{array}$ & $\begin{array}{l}\text { Specificity } \\
(95 \% \mathrm{Cl})\end{array}$ & $\begin{array}{l}\text { LR of positive } \\
\text { result }(95 \% \mathrm{Cl})\end{array}$ & $\begin{array}{l}\text { LR of negative } \\
\text { result }(95 \% \mathrm{Cl})\end{array}$ \\
\hline \multicolumn{9}{|c|}{ High-frequency oscillopsia test } \\
\hline Burgio et al. ${ }^{26}$ & 14 & 20 & 21 & 60 & $\begin{array}{c}40.0 \\
(0.24-0.58)\end{array}$ & $\begin{array}{c}75.0 \\
(0.64-0.84)\end{array}$ & $\begin{array}{c}1.60 \\
(0.92-2.79)\end{array}$ & $\begin{array}{c}0.80 \\
(0.59-1.08)\end{array}$ \\
\hline \multicolumn{9}{|c|}{ Hyperventilation-induced nystagmus } \\
\hline Robichaud et al. ${ }^{44}$ & 7 & & 31 & & $\begin{array}{c}18.4 \\
(0.08-0.34)\end{array}$ & & & \\
\hline \multicolumn{9}{|c|}{ Positional nystagmus test } \\
\hline Guidetti et al. ${ }^{32}$ & 34 & 0 & 494 & 133 & $\begin{array}{c}6.4 \\
(0.05-0.09)\end{array}$ & $\begin{array}{c}100 \\
(0.97-1.00)\end{array}$ & $\begin{array}{c}17.48 \\
(1.09-283.25)\end{array}$ & $\begin{array}{c}0.94 \\
(0.91-0.96)\end{array}$ \\
\hline \multicolumn{9}{|c|}{ Target condition = central vestibular dysfunction } \\
\hline \multicolumn{9}{|c|}{ Head-shaking nystagmus } \\
\hline Vicini et al. ${ }^{46}$ & 42 & 31 & 144 & 60 & $\begin{array}{c}22.6 \\
(0.17-0.29)\end{array}$ & $\begin{array}{c}65.9 \\
(0.55-0.76)\end{array}$ & $\begin{array}{c}0.66 \\
(0.45-0.98)\end{array}$ & $\begin{array}{c}1.17 \\
(0.99-1.39)\end{array}$ \\
\hline \multicolumn{9}{|l|}{ Head impulse test } \\
\hline Cnyrim et al. ${ }^{28}$ & 26 & 3 & 17 & 37 & $\begin{array}{c}60.5 \\
(0.44-0.75)\end{array}$ & $\begin{array}{c}92.5 \\
(0.80-0.98)\end{array}$ & $\begin{array}{c}8.06 \\
(2.64-24.58)\end{array}$ & $\begin{array}{c}0.43 \\
(0.29-0.63)\end{array}$ \\
\hline $\begin{array}{l}\text { Newman-Toker } \\
\text { et al. } .^{40}\end{array}$ & 31 & 0 & 3 & 8 & $\begin{array}{c}91.2 \\
(0.76-0.98)\end{array}$ & $\begin{array}{c}100 \\
(0.63-1.00)\end{array}$ & $\begin{array}{c}16.2 \\
(1.09-240.08)\end{array}$ & $\begin{array}{c}0.11 \\
(0.04-0.29)\end{array}$ \\
\hline \multicolumn{9}{|c|}{ Hyperventilation-induced nystagmus } \\
\hline Robichaud et al. ${ }^{44}$ & 14 & & 10 & & $\begin{array}{c}58.3 \\
(0.37-0.78)\end{array}$ & & & \\
\hline
\end{tabular}

population and in a theoretical primary care population are shown in Appendix 5 (available at www.cmaj.ca/cgi/content /full/cmaj.080910/DC1).

\section{Tests}

The performance of all studied tests and the analyses of tests described in a single study are presented in Appendix 6 (available at www.cmaj.ca/cgi/content/full/cmaj.080910 /DC1). Accurate evaluation of diagnostic tests should be based on the results of more than one study. Therefore, we describe four tests, all targeted for neuro-otologic conditions, that were evaluated in more than one study.

\section{Dix-Hallpike manoeuvre}

Both studies of the use of the Dix-Hallpike manoeuvre to diagnose benign paroxysmal positional vertigo used multiple vestibular tests as reference standards. Data from 114 patients were available, and we calculated a mean sensitivity of $80 \%$ (95\% confidence interval $[\mathrm{CI}] 71 \%-87 \%$ ).

\section{Head-shaking nystagmus test}

All nine studies of the use of the head-shaking nystagmus test used caloric measurement as part of the reference standard. Data from 4059 patients (eight studies) were available for pooled analysis (Figure 2 and Appendix 7, available at www .cmaj.ca/cgi/content/full/cmaj.080910/DC1). The pooled sensitivity was $45 \%$ (95\% CI $30 \%-62 \%$ ), the pooled specificity was $82 \%$ (95\% CI 68\%-90\%), the pooled positive likelihood ratio (LR) was 2.47 (95\% CI 1.48-4.14) and the pooled neg- ative LR was 0.67 (95\% CI 0.51-0.87). In these studies, the pooled probability of peripheral vestibular dysfunction after a positive head-shaking nystagmus test result increased from $27 \%$ to $48 \%$, and the probability after a negative result decreased from $27 \%$ to $25 \%$. Using an estimated prevalence of $33 \%$ for peripheral vestibular dysfunction in primary care patients with dizziness, ${ }^{7,9-11}$ the post-test probability of a positive head-shaking nystagmus test result was $55 \%$ and the post-test probability of a negative result was $25 \%$.

\section{Head impulse test}

The seven studies that evaluated the head impulse test for peripheral vestibular dysfunction used caloric measurement as a reference standard. Data were available from 869 patients (six studies) for pooled analysis (Figure 3 and Appendix 8, available at www.cmaj.ca/cgi/content/full/cmaj.080910/DC1). The pooled sensitivity was $63 \%$ (95\% CI $40 \%-81 \%)$, and the pooled specificity was $93 \%$ (95\% CI 83\%-98\%). The pooled positive LR was 9.29 (95\% CI 4.28-20.16), and the pooled negative LR was 0.40 (95\% CI 0.23-0.68). In these studies, the probability of peripheral vestibular dysfunction after a positive test result increased from $33 \%$ to $82 \%$, and the probability after a negative result decreased from $33 \%$ to $17 \%$. Because the estimated prevalence in primary care ${ }^{7,9-11}$ matched the prevalence in the studied population (both 33\%), the posttest probabilities were the same.

Two studies ${ }^{28,40}$ evaluated the accuracy of the head impulse test for central vestibular dysfunction in patients with vertigo. These studies used cranial magnetic resonance imaging as a 


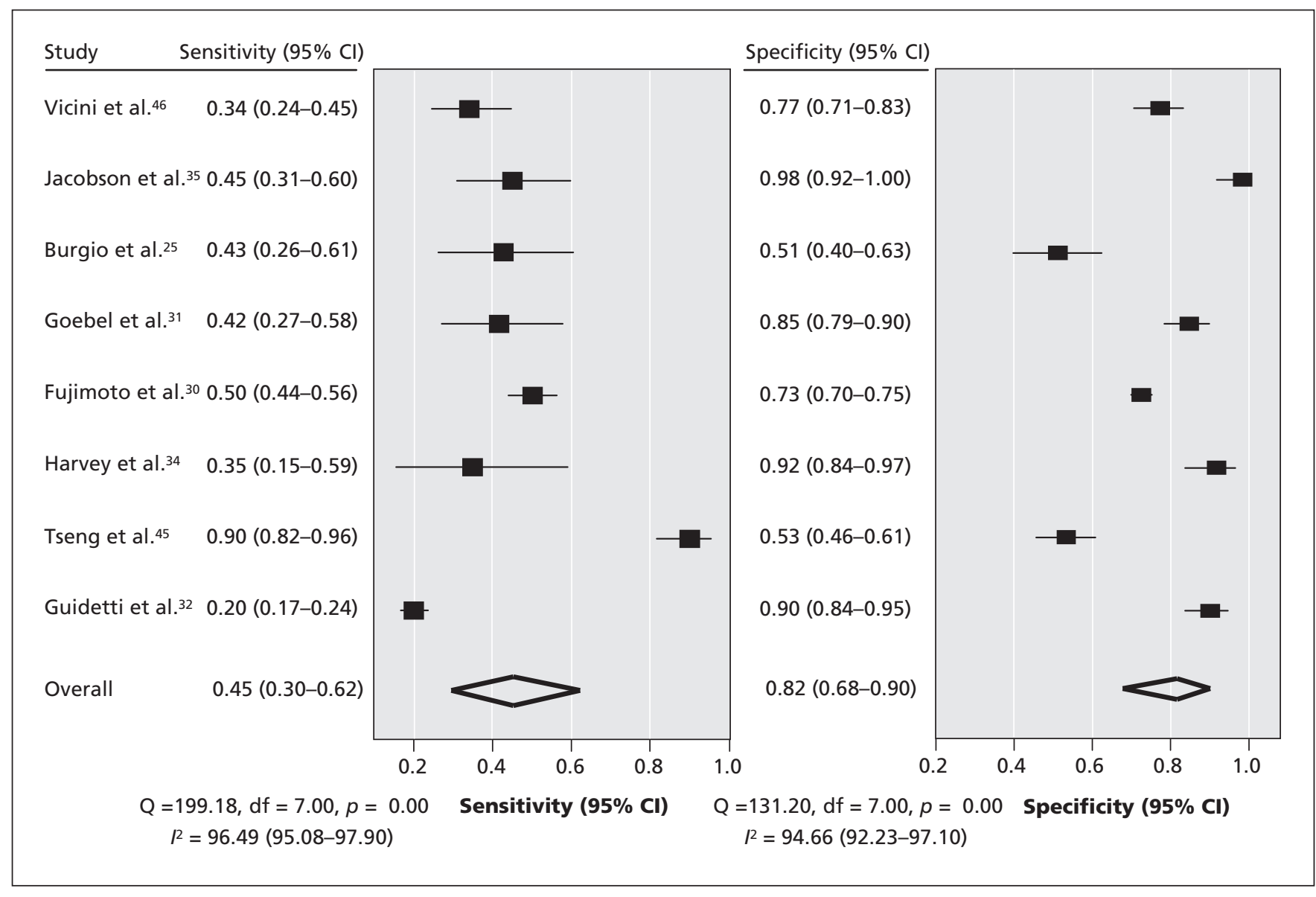

Figure 2: Sensitivity and specificity of the head-shaking nystagmus test for diagnosing unilateral vestibular dysfunction.

reference standard. Data from 125 patients were available for pooled analysis. A negative result of this test suggests a central lesion. The pooled sensitivity was 74\% (95\% CI 63\%-83\%), and the pooled specificity was $94 \%$ (95\% CI $83 \%-99 \%)$. The probability of central vestibular dysfunction after a positive head impulse test result decreased from $62 \%$ to $31 \%$, and the probability after a negative result increased from $62 \%$ to $95 \%$.

\section{Vibration-induced nystagmus test}

In the two studies that evaluated vibration-induced nystagmus, data were available from 463 patients, giving a calculated sensitivity of $84 \%$ (95\% CI $81 \%-88 \%$ ). We calculated specificity based only on one study, with a positive result of the vibration-induced nystagmus test increasing the probability of peripheral vestibular dysfunction from $13 \%$ to $59 \%$. Using the estimated prevalence of $33 \%$ for peripheral vestibular dysfunction in primary care patients with dizziness, ${ }^{7,-11}$ we estimated the post-test probability of a positive vibrationinduced nystagmus test result to be $83 \%$.

\section{Discussion}

The results of our review show that the empirical validation of commonly used diagnostic tests for dizziness in primary care is poor. We found few studies that focused on dizziness.
It was only possible to perform a meta-analysis of two tests in the neuro-otological field. Although many studies were performed for tests used in diagnosing dizziness, most were not diagnostic studies, were methodologically inadequate or did not include patients with dizziness.

All of the included studies used some type of preselection of patients and were intended to diagnose specific conditions, such as Ménière disease, peripheral vestibular dysfunction, major depressive disorder or panic disorder. The main interest in primary care, however, is the differentiation between selflimiting conditions, those for which adequate treatment is available and those that are dangerous or progressive or both and require referral or immediate treatment.

Although many tests are used to diagnose dizziness in primary care, we could not find any studies that included consecutive patients with dizziness. Studies about the use of patient history, pulse measurement, heart auscultation and balance in the diagnosis of dizziness are lacking.

Although we looked for studies of tests that are feasible in primary care, all of the included studies were, at least, partially conducted in secondary or tertiary settings. As a result, the high prevalence of the target conditions in the included populations (6\% for Ménière disease and $80 \%$ for vertigo) inflates the positive predictive values and decreases the negative predictive values. Furthermore, symptoms are probably 


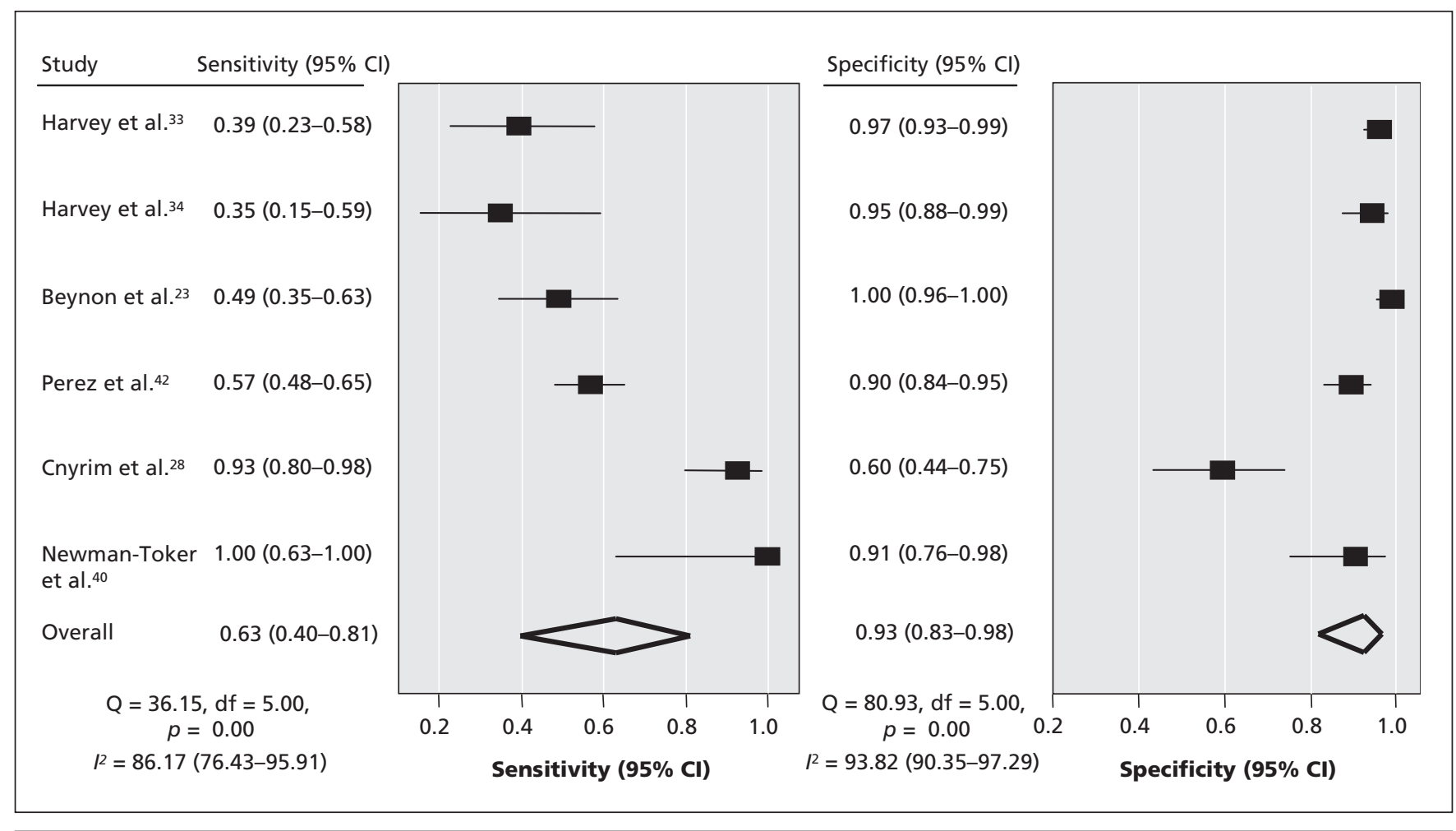

Figure 3: Sensitivity and specificity of the head impulse test for diagnosing unilateral vestibular dysfunction.

more severe or pronounced in patients referred for secondary or tertiary care, which may inflate both the sensitivity and specificity. Although we used prevalence in a hypothetical primary care population to estimate likelihood ratios, we could not correct for this phenomenon. This limits the generalizability of our results to patients in primary care. An exception may be the head-shaking nystagmus test and the head impulse test. The prevalence of peripheral vestibular dysfunction in primary care patients with dizziness was similar to that in the studied populations.

Although the prevalence of dizziness increases with age and the need for valid diagnostic tests or protocols is evident, especially for use in elderly patients, none of the studies in our review included an elderly population. As age increases, both the prevalence of dizziness and the risk of more serious causes of dizziness (stroke or cardiovascular diseases) increase. Comorbid conditions causing dizziness (e.g., diabetes, Parkinson disease) also become more prevalent.

Most tests for the diagnosis of dizziness were analyzed in a single study. The exceptions were the head-shaking nystagmus test, the head impulse test, the vibration-induced nystagmus test and the Dix-Hallpike manoeuvre. Promising tests within single test studies were the Vestibular Vertigo Questionnaire for the diagnosis of BPPV (positive LR 10.56, 95\% CI 3.54-31.5), the depression part of the Patient Health Questionnaire for the diagnosis of major depressive disorder (positive LR 12.51, 95\% CI 4.9631.53) and the panic disorder part of the Patient Health Questionnaire for the diagnosis of panic disorder (positive LR 25.1, 95\% CI 8.22-76.65).
Both the head-shaking nystagmus test and the head impulse test seem to be better suited for ruling in than for ruling out peripheral vestibular dysfunction. However, for conditions that are most often self-limiting (e.g., peripheral vestibular dysfunction), an increased probability from $27 \%$ to $48 \%$ after a positive test result is of limited value. The head impulse test performs better for the diagnosis of peripheral vestibular dysfunction, with an increased probability from $33 \%$ to $82 \%$ after a positive result and, based on two studies, an increased probability of central vestibular dysfunction from $62 \%$ to $95 \%$ after a negative result.

Many tests have been evaluated in patients without dizziness (e.g., peripheral neuropathy tests, visual acuity) or are included in the definition of the condition (e.g., hypoglycemia or orthostatic hypotension tests). Before the results of these studies can be used in the assessment of a certain diagnosis in a patient with dizziness, these tests must be validated in the appropriate domain. This is especially true for dizziness, because the cause is often not sufficiently supported by scientific evidence. In elderly patients, who often have more than one condition that can cause dizziness, physicians must be cautious in not treating these patients on the basis of test results that are not consistent with the patient's symptoms.

The appropriate patients to be included in studies of diagnostic tests for dizziness are those who present with dizziness as an isolated symptom and who are not at risk for the acutely life-threatening illnesses mentioned earlier. Prior selection of patients may affect test characteristics. The signs and symptoms must be described precisely, and the study design must comply with accepted methodologic criteria. ${ }^{49,50}$ 
Additional research into the tests that can be used in primary care to diagnose dizziness or to determine which patients need further testing or treatment is highly warranted.

\section{This article has been peer reviewed.}

Competing interests: None declared.

Contributors: All of the authors contributed to the conception and design of the study, the acquisition and interpretation of the data, and the drafting and revising of the manuscript. All of the authors approved the final version of the manuscript for publication.

Funding: The Netherlands Organisation for Health Research and Development provided funding for this review (grant no. 4200.0018). They had no role in the design of the study; data collection, analysis, or interpretation of the data; nor any role in the approval of publication of the finished manuscript.

\section{REFERENCES}

1. Colledge NR, Wilson JA, Macintyre CC, et al. The prevalence and characteristics of dizziness in an elderly community. Age Ageing 1994;23:117-20.

2. Kroenke K, Price RK. Symptoms in the community. Prevalence, classification, and psychiatric comorbidity. Arch Intern Med 1993;153:2474-80.

3. Sloane PD, Coeytaux RR, Beck RS, et al. Dizziness: State of the science. Ann Intern Med 2001;134(9 Pt 2):823-32.

4. van der Linden MW, Westert GP, de Bakker DH, et al. The second Dutch National Survey of General Practice. Utrecht/Bilthoven (Netherlands): NIVEL/RIVM; 2004.

5. Yardley L, Owen N, Nazareth I, et al. Prevalence and presentation of dizziness in a general practice community sample of working age people. Br J Gen Pract 1998;48: $1131-5$

6. Drachman DA, Hart CW. An approach to the dizzy patient. Neurology 1972;22: 323-34.

7. Hanley K, O'Dowd T. Symptoms of vertigo in general practice: a prospective study of diagnosis. Br J Gen Pract 2002;52:809-12.

8. Hoffman RM, Einstadter D, Kroenke K. Evaluating dizziness. Am J Med 1999;107: 468-78.

9. Kroenke K, Lucas CA, Rosenberg ML, et al. Causes of persistent dizziness. A prospective study of 100 patients in ambulatory care. Ann Intern Med 1992;117: 898-904.

10. Madlon-Kay DJ. Evaluation and outcome of the dizzy patient. J Fam Pract 1985; 21:109-13

11. Sloane PD, Dallara J, Roach C, et al. Management of dizziness in primary care. $J$ Am Board Fam Pract 1994;7:1-8.

12. Froehling DA, Silverstein MD, Mohr DN, et al. The rational clinical examination: Does this dizzy patient have a serious form of vertigo? JAMA 1994;271:385-8.

13. Tinetti ME, Williams CS, Gill TM. Dizziness among older adults: A possible geriatric syndrome. Ann Intern Med 2000;132:337-44.

14. EBM guidelines: evidence-based medicine. Vertigo (updated 2007 Nov.8) and BPPV (updated 2010 Apr.12). Cochrane Inside. Available: http://ebmg.wiley.com/ ebmg/ltk.koti

15. Brignole M, Alboni P, Benditt DG, et al. Task Force on Syncope ESoC. Guidelines on management (diagnosis and treatment) of syncope-update 2004. Europace 2004;6:467-537.

16. Verheij AA, van Weert HC, Lubbers WJ, et al. The guideline "Dizziness" of the Dutch College of General Practitioners. Huisarts Wet 2002;45:601-9.

17. Baloh RW. Approach to the evaluation of the dizzy patient. Otolaryngol Head Neck Surg 1995;112:3-7.

18. Bachmann LM, Coray R, Estermann P, et al. Identifying diagnostic studies in MEDLINE: reducing the number needed to read. J Am Med Inform Assoc 2002; 9:653-8.

19. Bachmann LM, Estermann P, Kronenberg C, et al. Identifying diagnostic accuracy studies in EMBASE. J Med Libr Assoc 2003;91:341-6.

20. Whiting P, Rutjes AW, Dinnes J, et al. Development and validation of methods for assessing the quality of diagnostic accuracy studies. Health Technol Assess 2004;8:iii:59-65.

21. Harbord RM, Deeks JJ, Egger M, et al. A unification of models for meta-analysis of diagnostic accuracy studies. Biostatistics 2007;8:239-51.

22. Reitsma JB, Glas AS, Rutjes AW, et al. Bivariate analysis of sensitivity and specificity produces informative summary measures in diagnostic reviews. J Clin Epidemiol 2005;58:982-90.

23. Beynon GJ, Jani P, Baguley DM. A clinical evaluation of head impulse testing Clin Otolaryngol Allied Sci 1998;23:117-22.

24. Brevern von M, Radtke A, Lezius F, et al. Epidemiology of benign paroxysmal positional vertigo: a population based study. J Neurol Neurosurg Psychiatry 2007;78: $710-5$

25. Burgio DL, Blakley BW, Myers SF. An evaluation of the head-shaking nystagmus test. Otolaryngol Head Neck Surg 1991;105:708-13.

26. Burgio DL, Blakley BW, Myers SF. The high-frequency oscillopsia test. J Vestib Res 1992;2:221-6.

27. Chan TP. Is benign paroxysmal positional vertigo underdiagnosed in hospitalised patients? Hong Kong Med J 2008;14:198-202.
28. Cnyrim CD, Newman-Toker D, Karch C, et al. Bedside differentiation of vestibular neuritis from central "vestibular pseudoneuritis". J Neurol Neurosurg Psychiatry 2008;79:458-60.

29. Cohen HS. Side-lying as an alternative to the Dix-Hallpike test of the posterior canal. Otol Neurotol 2004;25:130-4.

30. Fujimoto M, Rutka J, Mai M. A study into the phenomenon of head-shaking nystagmus: Its presence in a dizzy population. J Otolaryngol 1993;22:376-9.

31. Goebel JA, Garcia P. Prevalence of post-headshake nystagmus in patients with caloric deficits and vertigo. Otolaryngol Head Neck Surg 1992;106:121-7.

32. Guidetti G, Monzani D, Rovatti V. Clinical examination of labyrinthine-defective patients out of the vertigo attack: sensitivity and specificity of three low-cost methods. Acta Otorhinolaryngol Ital 2006;26:96-101.

33. Harvey SA, Wood DJ. The oculocephalic response in the evaluation of the dizzy patient. Laryngoscope 1996;106(1 Pt 1):6-9.

34. Harvey SA, Wood DJ, Feroah TR. Relationship of the head impulse test and headshake nystagmus in reference to caloric testing. Am J Otol 1997;18:207-13.

35. Jacobson GP, Newman CW, Safadi I. Sensitivity and specificity of the head-shaking test for detecting vestibular system abnormalities. Ann Otol Rhinol Laryngol 1990;99 (7 Pt 1):539-42.

36. Kentala E, Rauch SD. A practical assessment algorithm for diagnosis of dizziness. Otolaryngol Head Neck Surg 2003;128:54-9.

37. Lopez-Escamez JA, Lopez-Nevot A, Gamiz MJ, et al. Effectiveness of a structured questionnaire for diagnosis of Meniere's disease in the first visit. Acta Otorhinolaryngol Belg 2000;54:451-8.

38. Mandala M, Nuti D, Broman AT, et al. Effectiveness of careful bedside examination in assessment, diagnosis, and prognosis of vestibular neuritis. Arch Otolaryngol Head Neck Surg 2008;134:164-9.

39. Michel J, Dumas G, Lavieille JP, et al. Diagnostic value of vibration-induced nystagmus obtained by combined vibratory stimulation applied to the neck muscles and skull of 300 vertiginous patients. Rev Laryngol Otol Rhinol (Bord) 2001;122:89-94.

40. Newman-Toker DE, Kattah JC, Alvernia JE, et al. Normal head impulse test differentiates acute cerebellar strokes from vestibular neuritis. Neurology 2008;70:2378-85.

41. Norre ME. Diagnostic problems in patients with benign paroxysmal positional vertigo. Laryngoscope 1994;104:1385-8.

42. Perez N, Rama-Lopez J. Head-impulse and caloric tests in patients with dizziness. Otol Neurotol 2003;24:913-7.

43. Persoons P, Luyckx K, Desloovere C, et al. Anxiety and mood disorders in otorhinolaryngology outpatients presenting with dizziness: validation of the self-administered PRIME-MD Patient Health Questionnaire and epidemiology. Gen Hosp Psychiatry 2003;25:316-23.

44. Robichaud J, Desroches H, Bance M. Is hyperventilation-induced nystagmus more common in retrocochlear vestibular disease than in end-organ vestibular disease? $J$ Otolaryngol 2002;31:140-3.

45. Tseng HZ, Chao WY. Head shaking nystagmus: a sensitive indicator of vestibular dysfunction. Clin Otolaryngol Allied Sci 1997;22:549-52.

46. Vicini C, Casani A, Ghilardi P. Assessment of head shaking test in neuro-otological practice. ORL J Otorhinolaryngol Relat Spec 1989;51:8-13.

47. Weinberg J, Sade J. A simple and time saving cold mini caloric test. J Laryngol Otol 1984;98:701-5.

48. Whitney SL, Marchetti GF, Morris LO. Usefulness of the dizziness handicap inventory in the screening for benign paroxysmal positional vertigo. Otol Neurotol 2005;26:1027-33.

49. Bossuyt PM, Reitsma JB, Bruns DE, et al. The STARD statement for reporting studies of diagnostic accuracy: explanation and elaboration. Ann Intern Med 2003;138:W1-12.

50. Whiting P, Rutjes AW, Reitsma JB, et al. Sources of variation and bias in studies of diagnostic accuracy: a systematic review. Ann Intern Med 2004;140:189-202.

Correspondence to: Dr. Jacquelien Dros, Department of Family Medicine, Academic Medical Center, University of Amsterdam, Meibergdreef 15, 1105 AZ Amsterdam, the Netherlands;

j.dros@amc.uva.nl

\section{Additional information about the four tests studied in this article}

- Dix-Hallpike manoeuvre ${ }^{29}$ www.dizziness-and-balance.com/disorders/bppv/dix $\% 20$ hallpike.htm

- Head-shaking nystagmus ${ }^{34}$ www.dizziness-and-balance.com/research/hsn/Head Shaking Nystagmus.htm

- Head impulse test ${ }^{38}$ http://www.dizziness-and-balance.com/practice/head -impulse.html

- Vibration-induced nystagmus test ${ }^{38}$ www.dizziness-and-balance.com/practice/nystagmus /vibration_test.htm 